much a frontier in the study of planetary impact cratering.

We can look forward to further theoretical and experimental work on the interaction of strong blast waves with natural surfaces, perhaps supplemented with observations from old nuclearairburst and high-explosion tests. Theoretical studies (mostly numerical hydrocode computations) of impacts on a planet with an atmosphere are needed to understand how the meteorite's blast wave, the expanding plume of ejected debris, and the ambient atmosphere interact to produce the observed deposits.
Such processes are of minimal importance on planets with very thin atmospheres, such as Mars, but are much more important on the Earth and Venus. On Earth, however, fluvial erosion has erased most ejecta deposits of large impact craters. Venus, with its dense atmosphere and low erosion rates, promises to be a marvellous natural laboratory for the study of impact-atmosphere interactions.

H. J. Melosh is in the Lunar and Planetary Laboratory, University of Arizona, Tucson, Arizona 85721, USA.

\title{
BIOSENSORS
}

\section{NO release for good measure}

\section{Solomon H. Snyder}

NITRIC oxide is one of the most important messenger molecules in biology, mediating the killing by macrophages of tumour cells and bacteria, dilating blood vessels in response to neurotransmitters, and serving as a neurotransmitter in the brain. One of the major limitations in understanding these diverse roles of nitric oxide (NO) has been the difficulty in measuring it, as it is a free radical with a half-life of only a few seconds. On page 676 of this issue Malinski and Taha ${ }^{1}$ describe an unusual microsensor that can measure NO concentrations both at the external surface of single cells and in their interior, and which may eventually help provide a more detailed grasp of its physiological functions.

The first indication that nitric oxide functions as a biological mediator came from mice with a genetic deficiency of macrophages ${ }^{2}$. Nitrate excretion in the urine is normally increased in inflammatory conditions, but was absent in these mice; it was subsequently found that macrophages generate NO by oxidation of arginine using a nitric oxide synthase enzyme. Removal of arginine from the incubation medium, or the use of arginine derivatives that are NO synthase inhibitors, prevents macrophages from killing tumour cells and bacteria ${ }^{3}$.

It has been found that the relaxation of blood vessels by acetylcholine is impaired when the endothelium is removed, probably because the endothelial receptors respond to acetylcholine by generating an endothelial-derived relaxing factor (EDRF) which diffuses to the muscle layer to provoke relaxation ${ }^{4}$. The amount of NO released in this process is sufficient to account for the EDRF activity ${ }^{5}$.

In the brain the NO synthase enzyme is localized to discrete populations of neurons that are selectively resistant to neurotoxic damage in Huntington's dis- ease and stroke $^{6}$, and in the peripheral autonomic nervous system it is found in populations of neurons that regulate peristalsis in the intestine, in the nerves to the erectile tissue of the penis, and in neurons to the posterior pituitary and to the adrenal medulla. In these systems NO acts as a neurotransmitter: the synthase enzyme in the nerves causes NO release upon nerve stimulation. Inhibitors of NO synthase therefore block physiological responses. Of the three forms of NO synthase that have so far been cloned, the neuronal ${ }^{7}$ and endothelial ${ }^{8,9}$ enzymes bind calmodulin and are activated by calcium, but the macrophage enzyme $\mathrm{e}^{10-12}$ is activated by synthesis of new enzyme in response to immune stimulation and is insensitive to calcium. Nitric oxide synthase is one of the most sensitive enzymes in biology, in keeping with its responsibility for finetuning the levels of a major physiological mediator. The development of a sensor that can rapidly respond to minute changes in concentration of NO in situ is therefore a considerable technical advance towards monitoring the release and distribution of $\mathrm{NO}$ in the body.

In designing their microsensor, Malinski and Taha have taken advantage of the ease of oxidation of $\mathrm{NO}$ and its ready diffusibility ${ }^{1}$. It was already known that highly conducting polymeric metalloporphyrins can catalyse the electrochemical oxidation of small molecules. The authors created a polymer of porphyrin with nickel as the central metal. As the polymer also reacts with NO-related anions such as nitrites, they coated the polymer with Nafion, a substance that is permeable to NO but blocks the diffusion of nitrite.

The resultant electrochemical detector is highly sensitive and specific. It detects a concentration of $\mathrm{NO}$ of about $10 \mathrm{nM}$ in solution. In a volume equivalent to a single cell, it can readily detect $10^{-20}$ mol of NO, which is substantially less than the amount of NO produced in individual cells. The authors applied their method to measuring NO in blood vessels and, in the process, established definitively that NO is endothelialderived relaxing factor. This factor is released by endothelial cells in response to molecules that dilate blood vessels, such as acetylcholine and bradykinin, and diffuses from the endothelial cells to the adjacent smooth muscle cells, causing them to relax so that the blood vessel dilates ${ }^{4}$. When the microsensor is placed on the surface of endothelial cells in the aorta, it detects substantial levels of NO released into the medium after the administration of bradykinin. Implantation of the microsensor into a nearby smooth muscle cell reveals that NO concentrations have increased in response to bradykinin.

The NO microsensor will help to probe several unresolved features of NO function. When activated, both macrophages and neutrophils generate NO (ref. 3), which before it kills tumour cells and bacteria presumably hits lymphocytes and other cells in the vicinity of the inflammatory response. Does NO affect these other cells? The sensor should be able to detect NO in any such cellular targets. Nitric oxide synthase occurs in selected neuronal populations in which NO seems to act as a neurotransmitter ${ }^{6}$; the sensor may reveal whether firing of these neurons increases NO only in adjacent neurons or whether glia and other cells are also affected. It may be that NO is a participant in models of synaptic plasticity, such as long-term potentiation and long-term depression, because inhibitors of NO synthase interfere with these processes ${ }^{6}$. Intracellular monitoring of $\mathrm{NO}$ concentrations could also clarify the sources and targets of $\mathrm{NO}$ in these instances.

Solomon H. Snyder is in the Department of Neuroscience, Johns Hopkins University School of Medicine, 725 N. Wolfe Street, Baltimore, Maryland 21205, USA.

1. Malinski, T. \& Taha, Z. Nature 358, 676-678 (1992).

2. Stuehr, D. J. \& Marletta, M. A. Proc. natn. Acad. Sci. U.S.A. 82, 7738-7742 (1985)

3. Nathan, C. F. \& Hibbs, J. B. Jr Curr. Opin. Immun. 3. 65-70 (1991).

4. Furchgott, R. F. \& Zawadzki, J. V. Nature 288, 373-376 (1980).

5. Palmer, R. M. J., Ferridge, A. G. \& Moncada, S. Nature 327, 524-526 (1987)

6. Bredt. D. S. \& Snyder, S. H. Neuron 8, 3-11 (1992)

7. Bredt, D. S. et al. Nature 352, 714-718 (1991).

8. Lamas, S., Marsden, P. A., Li, G. K., Tempst, P. \& Michel, T. Proc. natn. Acad. Sci. U.S.A. 89, 6348-6352 (1992)

9. Janssens, S. P., Shimouchi, A., Quertermous, T., Bloch,

D. B. \& Bloch, K. D J biol Chem 267, 14519-14522 (1992)

10. Lyons, C. R., Orloff, G. J. \& Cunningham, J. M. J. biol. Chem. 267, 6370-6374 (1992).

11. Xie, Q. W. et al. Science 256, 225-228 (1992) 12. Lowenstein, C. J., Glatt, C. S., Bredt, D. S. \& Snyder. S. H. Proc. natn. Acad. Sci. U.S.A. (in the press). 